Pyloric atresia is a rare congenital anomaly; to date, only 29 newborns with this defect have been reported. Though familial occurrence of this anomaly has been reported twice (Benson and Coury, 1951; Thompson et al, 1968; Bronsther, Nadeau, and Abrams, 1971), no previous comments have been made concerning a possible genetic aetiology.

The purpose of this paper is to report 5 additional familial cases* from two unrelated Israeli sibships and to stress the genetic factor responsible for this anatomical defect.

\section{Family A.}

This is a Jewish family (Fig. 1) from Iraq. The parents of the affected patients are first cousins. The family pedigree shows 2 infant deaths in the affected sibship, 5 deaths in the father's, and 3 in the mother's sibships while the family was still in Iraq. No reliable information could be obtained regarding the causes of death or the ages of the deceased.

Case 1. III.9, a male, was born at term following a normal pregnancy except for maternal hydramnios. Vomiting of gastric contents started immediately after birth. A plain $x$-ray of the abdomen showed a dilated stomach ('one bubble') with no gas beyond, suggestive of pyloric obstruction. At operation the stomach was markedly dilated with thickened walls. A fibrotic band, $3 \mathrm{~cm}$ long, connecting the stomach to the first part of the duodenum, replaced the pylorus. A Billroth I operation was carried out, but the infant died soon after operation.

Case 2. III.11, a female, was born at term. Maternal hydramnios was recorded. Vomiting with abdominal distension appeared on the 3rd day, followed by dyspnea and signs of dehydration. A plain film of the abdomen showed air under the diaphragm. At operation, a 6-cm rent on the posterior wall of the stomach was found, with a large amount of milk in the peritoneal cavity. The pylorus was atretic with a gap of $1 \mathrm{~cm}$ be-

\footnotetext{
Received 20 July 1971.

* Three were operated upon in our service and two were treated elsewhere.
}

tween the stomach and duodenum. The tear was closed and an anterior gastrojejunostomy was carried out. The infant died 14 hours after operation. Severe peritonitis was found at necropsy.

Case 3. III.14, a female, was also born after a pregnancy with hydramnios. Frothy sputum and vomiting appeared 2 days after birth. Radiology of the oesophagus and stomach showed complete obstruction of the gastric outlet. At operation a fibrotic band, $2.5 \mathrm{~cm}$ long, connected the stomach to the duodenum. End-toside anastomosis between the antrum and the second part of the duodenum was performed. The postoperative course was uneventful and the child has been well to date, 2 years after the operation.

\section{Family Ch.}

This is also a Jewish family (Fig. 1) from Iraq, but not related to the previous one. The patients' parents are not related. No other cases of infant death are known in the family and this appears to be the only affected sibship in the family at large.

Case 4. III.4, a female premature, weighed $2100 \mathrm{~g}$ at birth. She started vomiting gastric contents on the 2nd day of life when she also developed jaundice and respiratory distress with episodes of apnoea. She was operated upon at the age of 5 days, following radiological evidence of a 'single bubble' and no air beyond the pylorus. A double membranous type of atresia was found and excised through a gastrostomy. Postoperative vomiting produced an aspiration pneumonia resulting in the patient's death on the 9th day. In search for a cause for the postoperative vomiting, no residual pyloric obstruction was found at necropsy.

Case 5. III.5, a male infant, was born after a pregnancy with hydramnios. From birth the baby was in respiratory distress with frequent episodes of apnoea requiring resuscitation. Concomitantly, he had bouts of vomiting of gastric contents. Radiology of the oesophagus and stomach showed complete obstruction of the pylorous. At operation, a fibrotic band, $2 \mathrm{~cm}$ long, connected the stomach to the first part of the duodenum. A retrocolic gastrojejunostomy was performed. Respiratory distress and apnoeic spells continued and he died a 


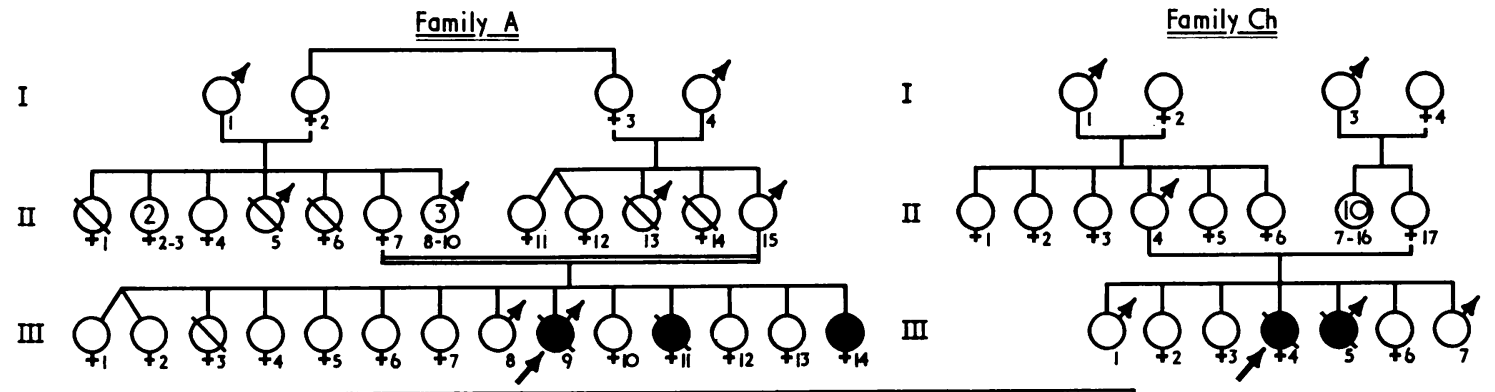

$O$ =normal $Q$ =offected $Q$ =death in infancy (cause unknown) $Q$ =death from pyloric atresio

Fig. 1. Pedigrees of Family A and Family Ch.

few hours after the operation. At necropsy hyaline membrane disease was found.

\section{Discussion}

The clinical picture of non-bilious vomiting in a newborn with a history of maternal hydramnios is characteristic of pyloric atresia. Only the upper abdomen is distended and a small amount of meconium may be passed. A plain film of the abdomen shows a typical 'single bubble' in the stomach with no air distally. The use of contrast media is not necessary for establishing the diagnosis.

Delay in diagnosis of this rare anomaly may be partly due to the absence of bile in the vomit. Distinction from hypertrophic pyloric stenosis, which clinically resembles pyloric atresia, is based on time of the appearance of symptoms. Delay in diagnosis and such complications as aspiration pneumonia, additional congenital anomalies, or hyaline membrane disease as observed in one of our cases, account for the poor results of treatment. The only survivor among our 5 patients was operated upon on the 2nd day of life and had no other complications.

Two anatomical types of pyloric atresia are known: absence of continuity of the pyloric wall (found so far in 9 patients) and complete membranous obstruction of the gastric outlet (found in 20 patients).

The two anatomical types present an identical clinical picture. The surgical treatment, however, is different: while a diaphragm can be excised through a gastrostomy, a fibrotic band should be by-passed by means of a gastroduodenostomy or a gastrojejunostomy.

In considering the aetiology of this anomaly, most authors have approached it from anatomic or embryologic view points. Tandler (1900) attributed it to be the failure of recanalization of the gut. Later studies by Barnard and Louw (1956) and Santulli and Blanc (1961), challenged Tandlers' theory and suggested mechanical and vascular injury as causative factors resulting in atresia of the affected portions of the gut. Although 2 affected families have been previously reported genetic determination of this anomaly was not considered. With our series the number of familial cases amounts to 9 out of a total of 34 reported. This strongly suggests a genetic determination. The following points are relevant in substantiation of a postulated mode of inheritance: (1) No known parent to child transmission; (2) consanguinity among parents; (3) an equal sex distribution among affected patients ( 13 boys and 18 girls);* (4) a $1: 3$ ratio of affected to non-affected patients (Table I). All those support an autosomal recessive mode of inheritance.

TABLE I

\begin{tabular}{l|c|c|c}
\hline \multicolumn{1}{c|}{ Authors } & Sibship Size & \multicolumn{2}{|c}{ No. of Affected Sibs } \\
\cline { 2 - 4 } & & Observed & Expected \\
\hline Bronsther et al (1971) & 5 & 2 & 1.64 \\
Present (family A.) & 14 & $3+$ & 3.56 \\
Present (family Ch.) & 7 & 2 & 2.02 \\
\hline Total & 26 & 7 & $7 \cdot 22 \ddagger$ \\
\hline
\end{tabular}

* Of 4 reported families only 3 where the sibship size was known are included.

$t$ The number of diagnosed cases. There may have been more as indicated in the family history.

₹ The expected number calculated by the Lenz-Hogben method on the assumption of complete asertainment.

Further testing of this genetic hypothesis should include analysis of the data to determine whether the proportion of families with multiple cases, relative to those with single cases does not deviate from the expected. This, however, is impossible to calculate without information about families of all cases, which is lacking.

We believe that the data, limited as they are, permit the tentative conclusion that pyloric atresia is

* The sex of 3 cases was not stated. 
genetically determined and transmitted as an autosomal recessive disease. The two different anatomic variants of this anomaly were observed twice in the same sibships (Bronsther et al, 1971; Thompson et al, 1968). How 2 parents, presumably heterozygous for the same mutant gene, can produce 2 affected offspring with different anatomic defects, remains to be explained. It is of interest that the only family reported, in which the anatomical lesion in 3 affected sibs was identical, is our family A., in which the parents are first cousins. Here, the mutation transmitted to both parents from a common ancestor is most likely to be identical. In addition, this couple shares many more identical genes than any unrelated couple, thereby contributing to more homogeneity among their offspring.

\section{Summary}

Five familial cases of pyloric atresia are reported. Three cases from one sibship were the offspring of a first cousin marriage. Two were born to unrelated parents. With 2 affected families, formerly re- ported, this series raises the number of familial cases to 9 out of a total of 34 cases recorded so far. The high incidence of familial cases in such a rare anomaly, suggests genetic determination. An almost equal sex distribution among the affected, born to healthy parents, with one example of consanguinity among the four reported families and a 1:3 ratio of affected to non-affected sibs, indicate an autosomal recessive mode of inheritance.

\section{REFERENCES}

Barnard, C. N. and Louw, J. H. (1956). The genesis of intestinal atresia. Minnesota Medicine, 39, 745.

Benson, C. D. and Coury, J. J. (1951). Congenital intrinsic obstruction of the stomach and duodenum in the newborn. Archives of Surgery, 62, 856-866.

Bronsther, B., Nadeau, M. R., and Abrams, M. W. (1971). Congenital pyloric atresia: a report of three cases and review of the literature. Surgery, 69, 130-136.

Santulli, T. V. and Blanc, W. A. (1961). Congenital atresia of the intestine: Pathogenesis and treatment. Annals of Surgery, 154, 939-948.

Tandler, J. (1900). Zur Entwicklungsgeschichte des menschlichen Duodenum im frühen Embryonal Stadien. Gegenbaur's morphologisches fahrbuch, 29, 187-216.

Thompson, N. W., Parker, W., Schwartz, S., and Holt, J. F. (1968). Congenital pyloric atresia. Archives of Surgery, 97, 792-796. 